\title{
Ultrasound Monitoring of Structural Urinary Tract Disease in Schistosoma haematobium Infection
}

\author{
Charles H King
}

Division of Geographic Medicine, W137, Case Western Reserve University SOM, 10900 Euclid Avenue, Cleveland, Ohio 44106-4983, USA

\begin{abstract}
A major advance in our understanding of the natural history of Schistosoma haematobium-related morbidity has come through the introduction of the portable ultrasound machines for non-invasive examination of the kidneys and bladder. With the use of generators or battery packs to supply power in non-clinical field settings, and with the use of instant photography or miniaturized thermal printers to record permanent images, it is possible to examine scores of individuals in endemic communities every day. Broad-based ultrasound screening has allowed better definition of age-specific disease risks in urinary schistosomiasis. Results indicate that urinary tract abnormalities are common (18\% overall prevalence) in $\mathrm{S}$. haematobium transmission areas, with a $2-4 \%$ risk of either severe bladder abnormality or advanced ureteral obstruction. In longitudinal surveys, ultrasound studies have shown that praziquantel and metrifonate therapy are rapidly effective in reversing urinary tract abnormalities among children. The benefits of treating adults are less well known, but research in progress should help to define this issue. Similarly, the prognosis of specific ultrasound findings needs to be clarified, and the ease of sonographic examination will make such long-term follow-up studies feasible. In summary, the painless, quick, and reproducible ultrasound examination has become an essential tool in the study of urinary schistosomiasis.
\end{abstract}

Key words: ultrasonography - diagnostic imaging - schistosomiasis haematobia - hydronephrosis - bladder diseases

Urinary schistosomiasis afflicts an estimated 111 million persons in Africa and the Middle East (van der Werf \& de Vlas 2001). Although Schistosoma haematobium infection may sometimes result in only minimal pathology, there are a significant proportion of infected patients (25-50\%) who experience moderate to severe morbidity (King et al. 1988). Population surveys in endemic areas indicate that hematuria, dysuria, anemia, and inflammatory urinary tract pathology are the most common forms of S. haematobium-associated disease (Chen \& Mott 1989). Urinary schistosomiasis typically manifests as a lifelong process of tissue injury because, although individual $S$. haematobium worms survive only $4-5$ years, multiple worm infections (and reinfection) are common in high-risk areas, so that parasite-related inflammation and fibrosis continue for decades. After 20 to 30 years of infection, the late complications of S. haematobium infection begin to emerge clinically. These more life-threatening, late disorders include kidney dysfunction, obstruction of the ureters or bladder outflow, and/or urothelial metaplasia and cancer formation (King 2001).

With the advent of safe and effective oral therapy, repeated drug treatment has become the favored approach to controlling disease due to urinary schistosomiasis

This work was supported by grants from the National Institutes of Health (AI45473, TW/ES01543), the Edna McConnell Clark Foundation, and the Rockefeller/WHO-TDR Joint Funding Venture.

Fax: +1-216-368-4825. E-mail: chk@ po.cwru.edu

Received 18 June 2002

Accepted 15 August 2002
(WHO 1993). The aim of this therapy is to reduce infection intensity and thereby minimize (or even prevent) tissue damage and risk for disease. Given the expense of drug delivery on a population basis, certain operational questions arise: i.e., What is the optimal timing of drug treatment to prevent or regress structural morbidity? and Are there persons at special risk for morbidity, for whom more intensive therapy should be provided?

Because structural morbidity caused by $S$. haematobium is readily demonstrated on ultrasound examination, the sonogram has become a very useful tool for defining the population prevalence of infection-associated disease. Ultrasound-based population surveys have permitted an effective assessment of the reversibility of structural disorders among different age groups, and they are beginning to provide the essential data for decision analysis on the choice of treatment regimens for longterm population-based drug treatment programs.

\section{MATERIALS AND METHODS}

The findings summarized in this paper are derived from cross-sectional and longitudinal morbidity surveys performed in Coast Province, Kenya, primarily in the Msambweni area of Kwale District. This area is highly endemic for S. haematobium, but does not support transmission of other forms of schistosomiasis, i.e., S. mansoni or S. intercalatum. Details of these studies, performed during the years 1984-1992, 1997, and 2000-present, have been previously published (King et al. 1988, 1991, 1992, Olds et al. 1999, Subramanian et al. 1999, Hodder et al. 2000). Target study populations have comprised: (a) school age children from area villages and (b) total populations of selected villages within the study area. All participants in these studies gave informed consent and were studied under protocols approved by the Human 
Investigation Review Boards of University Hospitals of Cleveland, Ohio, USA, and of the Kenya Medical Research Institute (KEMRI), Nairobi, Kenya.

Ultrasonographic examinations were performed in the field with the use of portable ultrasound machines powered by generator (initially Toshiba Sonolayer SL, later Shimadzu model SDU-350A) or a rechargeable battery pack (Sonosite 180). Urinary tract pathology was detected by sector scanning and scored according to standardized protocols (Subramanian et al. 1999, Richter et al. 2000). Urinary tract imaging was performed after adequate hydration with the bladder full. Post-voiding images were also obtained for those subjects demonstrating kidney, ureteral, or bladder abnormalities. Images were stored for later review, either with the use of Polaroid cameras, portable thermal printers, or by image capture in electronic files.

\section{RESULTS AND DISCUSSION}

In $S$. haematobium infection, the adult schistosomes preferentially localize to the veins of the kidneys, ureters and bladder, although other organs of the pelvic area are often involved (Smith \& Christie 1986, Poggensee et al. 1999). Egg deposition by mature female worms induces local inflammation, with granulomas, polyps, ulcers, sandy patches, and calcification of the walls of the ureters, bladder and reproductive organs (Fig. 1). Hydronephrosis frequently occurs, either because of acute inflammation localized in the ureteral wall, or as a late consequence of cumulative fibrotic injury to the ureters (Smith \& Christie 1986). In studies of S. haematobium-related morbidity from different geographic areas, results of ultrasound studies have been generally well correlated with findings from IVP and cystoscopic examination. However, some radiologists feel that there are significant differences in the information provided by IVP versus ultrasound examination: tissue calcification and ureteral dysfunction are likely to be more apparent on dye-contrast roentgenographic studies, while hydronephrosis and deformity of poorly-

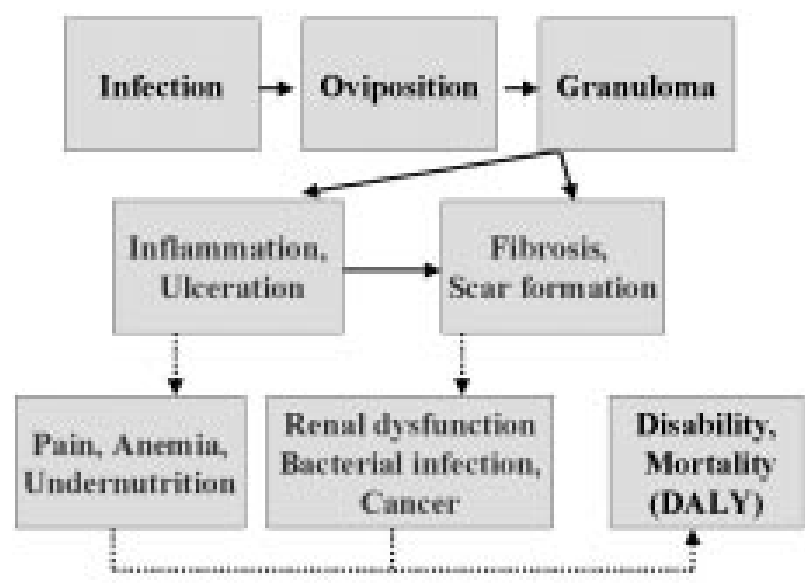

Fig. 1: probable disease causation pathway for urinary schistosomiasis due to Schistosoma haematobium infection. Solid lines indicate links based on experimental evidence. Dotted lines indicate associations where there is suggestive but not conclusive evidence of causation. function kidneys are better assessed on ultrasound examination.

Cross-sectional surveys utilizing ultrasonography in endemic villages in coastal Kenya indicate a $15-22 \%$ overall prevalence of bladder thickening and granulomata, with peak prevalence (36\%) between the ages of 16 and $20 \mathrm{yrs}$, one to five years after the peak of infection intensity (Fig. 2). Hydronephrosis is found in 5\% of residents, with peak prevalence of $10 \%$ among adolescents aged 12 15 , with a second peak $(6 \%)$ among subjects older than 49 yrs. It appears from longitudinal treatment studies that "acute" polypoid-granulomatous lesions, more common in younger individuals, are first to regress after therapy, and may disappear within weeks after treatment (King et al. 1992, Hatz et al. 1998). "Late" complications, such as hydronephrosis, may take a longer time (1-2 yrs) to show improvement, particularly in males (King et al. 1992). Elimination of infection does not terminate ongoing immune response to eggs trapped within tissues, and it is possible that fibrosis progresses for some time after successful cure of infection, and this may result in persistent dysfunction of the urinary tract.

In high transmission areas, reinfection with $S$. haematobium may occur in 1-2 yrs, with resulting recrudescence of urinary tract abnormalities detected by ultrasound (Hatz et al. 1998). This observation indicates the need for repeated antiparasitic therapy during the highest risk period of life, i.e., childhood and early adolescence, in order to minimize risk for late complications of urinary schistosomiasis. Analysis of compliance and drug efficacy of an eight year control project in coastal Kenya

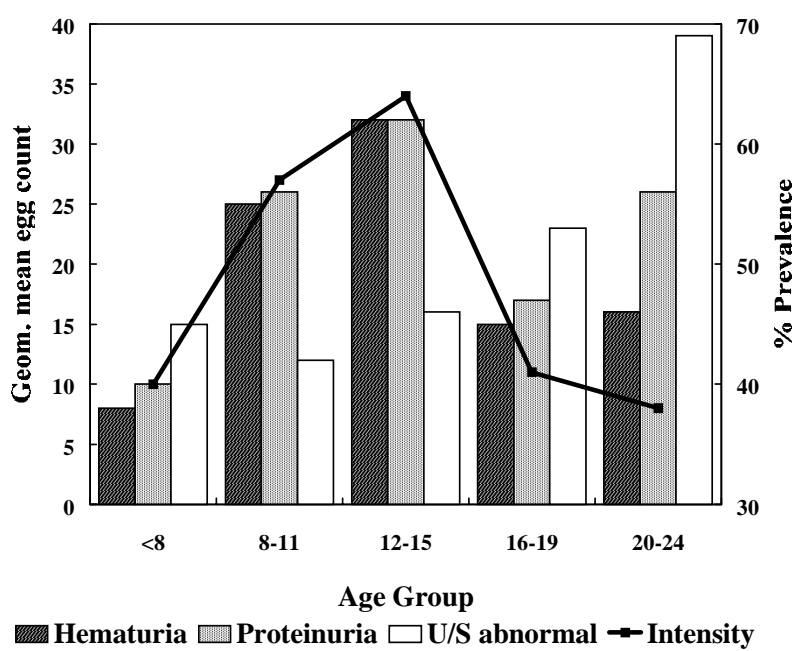

Fig. 2: contrasting findings for age-specific infection and disease in urinary schistosomiasis in the Msambweni area of Coast Province, Kenya. The dark line indicates geometric mean intensity of infection prior to first treatment for the age groups indicated. Note peak intensity of infection in the 12-15 yrs age group. Shaded and open bars indicate group-specific prevalence of infection-associated morbidity in terms of hematuria, proteinuria, or structural abnormalities of the bladder or kidneys as detected on ultrasound. Although peak prevalence of hematuria and proteinuria mirrors the infection intensity profile, ultrasound abnormalities become more common only in older age groups (>15 yrs), 1-5 yrs after the greatest period of Schistosoma haematobium infection intensity. 
indicates that regular treatment of school age children can result in a $>50 \%$ reduction in an individual's cumulative experience with parasite infection and his or her exposure to egg deposition and consequent disease (Fig. 3, see also Subramanian et al. 1999).

Does treatment during childhood have prolonged effects on the risk for S. haematobium-related morbidity? When young adults are reexamined 7-13 yrs after an antischistosomal treatment campaign, they have fewer bladder abnormalities and a reduced number of cases of hydronephrosis when compared to age- and gendermatched controls from untreated villages (Subramanian et al. 1999). This is despite of the fact that, at the time of their follow up examinations, the previously treated subjects have about the same prevalence and intensity of $S$. haematobium infection as untreated controls.

There remain, however, unanswered questions about the clinical implications and prognosis of urinary schistosomiasis-related abnormalities detected on ultrasound. Are the abnormalities observed among older adults reversible with therapy? What is the association between structural urinary tract abnormalities and renal insufficiency, risk for bacterial superinfection, and cancer formation? and finally, How do sonographic findings predict disability levels for currently- or previously-infected indi-

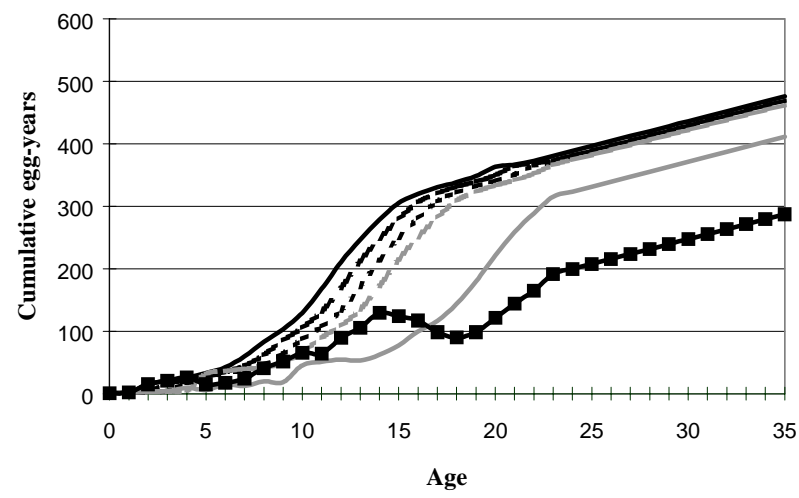

\begin{tabular}{|c|c|}
\hline $\begin{array}{l}\text { - in } 1984 \text { pre-Rx } \\
\text { - 'after } 2 \text { years } \\
\text { in } 1992 \text { after } 8 \text { years }\end{array}$ & $\begin{array}{l}\text { - -after } 1 \text { year } \\
\text { - "after } 3 \text { years } \\
\rightarrow-\text { projected 1997-worst case }\end{array}$ \\
\hline
\end{tabular}

Fig. 3: effects of repeated therapy on cumulative egg burden in an area of high prevalence of Schistosoma haematobium Lines indicate the average cumulative egg-year experience for individuals aged 0-35 in the Msambweni area of Coast Province, Kenya, during an annual drug treatment campaign for urinary schistosomiasis. Values are calculated from measured infection intensities at each age for age-cohorts followed during the course of the study (19841992). With repeated annual treatment, the egg-experience curve shifts rightward due to suppression of infection. Note that several years of therapy are required to significantly reduce egg-experience in age groups over 10. By the eighth year of the program (1992), the greatest impact is noted for 14- and 15-yrs olds, who had participated throughout the time of peak childhood transmission. The solid line with squares indicates the projected egg-experience for different age groups as of 1997, 5 yrs after cessation of treatment, assuming a return of pretreatment community levels of transmission after $2 \mathrm{yrs}$ without treatment. Note the continuing benefits for older, previously treated subjects who have reached age 20-25 yrs. viduals? Answers to some of these questions are expected from longitudinal studies now in progress, but more work will be needed to fully understand the natural history of treated and untreated schistosomiasis haematobia.

In summary, ultrasound examination provides a painless, quick, and reproducible measure of the structural urinary tract morbidity associated with $S$. haematobium infection. Its introduction permitted, for the first time, an evaluation of $S$. haematobium-associated disease on a population basis, and it is clear that the portable ultrasound has now become an essential tool in field studies of urinary schistosomiasis.

\section{ACKNOWLEDGEMENTS}

To Dr Eric Muchiri, Dr John Ouma, and Mr Peter Mungai of the Division of Vector Borne Diseases, Ministry of Health (Kenya) and Dr Davy Koech of the Kenya Medical Research Institute for their invaluable contributions to the studies outlined in this review. To Dr Jagon Muruka, Dr Philip Magak, Mr Edmund Ireri, and Ms Hilda Kadzo of the Dept. of Radiology, University of Nairobi, and Dr Ruth Greenblatt and Dr Patrick Bryan, formerly of CWRU, for their essential role in performing and analyzing the many thousands of field ultrasound examinations that form the basis of this paper.

\section{REFERENCES}

Chen MG, Mott KE 1989. Progress in assessment of morbidity due to Schistosoma haematobium infection. Trop Dis Bull 86: R1-R36.

Hatz CF, Vennervald BJ, Nkulila T, Vounatsou P, Kombe Y, Mayombana C, Mshinda H, Tanner M 1998. Evolution of Schistosoma haematobium-related pathology over 24 months after treatment with praziquantel among school children in Southeastern Tanzania. Am J Trop Med Hyg 59: 775-781.

Hodder SL, Mahmoud AAF, Sorenson K, Weinert DM, Stein RL, Ouma JH, Koech D, King CH 2000. Predisposition to urinary tract epithelial metaplasia in Schistosoma haematobium infection. Am J Trop Med Hyg 63: 133-138.

King CH 2001. Disease in schistosomiasis haematobia. In AAF Mahmoud, Schistosomiasis, Imperial College Press, London, p. 265-296.

King CH, Keating CE, Muruka JF, Ouma JH, Houser H, Siongok TK, Mahmoud AAF 1988. Urinary tract morbidity in schistosomiasis haematobia: associations with age and intensity of infection in an endemic area of Coast Province, Kenya. Am J Trop Med Hyg 39: 361-368.

King CH, Lombardi G, Lombardi C, Greenblatt R, Hodder S, Kinyanjui H, Ouma J, Odiambo O, Bryan PJ, Muruka J, Magak P, Weinert D, Mackay W, Ransohoff D, Houser H, Koech D, Siongok TK, Mahmoud AAF 1988. Chemotherapy-based control of schistosomiasis haematobia. I. Metrifonate versus praziquantel in control of intensity and prevalence of infection. Am J Trop Med Hyg 39: 295-305.

King CH, Muchiri E, Ouma JH, Koech D 1991. Chemotherapybased control of schistosomiasis haematobia. IV. Impact of repeated annual chemotherapy on prevalence and intensity of Schistosoma haematobium infection in an endemic area of Kenya. Am J Trop Med Hyg 45: 498-508.

King CH, Muchiri EM, Ouma JH 1992. Age-targeted chemotherapy for control of urinary schistosomiasis in endemic populations. Mem Inst Oswaldo Cruz 87: 203-210.

Olds GR, King CH, Hewlett J, Olveda R, Wu G, Ouma JH, Peters PA, McGarvey S, Koech D, Liu CY, Aligui G, Gachihi G, Kombe Y, Parraga I, Ramirez B, Whalen C, Horton RJ, 
Reeve P 1999. Double-blind placebo controlled study of concurrent administration of albendazole and praziquantel in school children with schistosomiasis and geohelminths. J Infect Dis 179: 996-1003.

Poggensee G, Feldmeier H, Krantz I 1999. Schistosomiasis of the female genital tract: public health aspects. Parasitol Today 15: 378-381.

Richter J, Hatz C, Campagne G, Bergquist NR, Jenkins JM 2000. Ultrasound in Schistosomiasis: a Practical Guide to the Standardized Use of Ultrasonography for the Assessment of Schistosomiasis-related Morbidity, World Health Organization, Geneva, $49 \mathrm{pp}$.

Smith JH, Christie JD 1986. The pathobiology of Schistosoma haematobium infection in humans. Hum Pathol 17: 333345.

Subramanian AK, Mungai P, Ouma JH, Magak P, King CH, Mahmoud AAF, King CL 1999. Long-term suppression of adult bladder morbidity and severe hydronephrosis following selective population chemotherapy for Schistosoma haematobium. Am J Trop Med Hyg 61: 476-481.

van der Werf MJ, de Vlas SJ 2001. Morbidity and Infection with Schistosomes or Soil-transmitted Helminths, Erasmus University, Rotterdam, p. 1-103.

WHO-World Health Organization 1993. The Control of Schistosomiasis: Second Report of the WHO Expert Committee, World Health Organization, Geneva. 